\title{
Production et qualité nutritionnelle des épis de maïs frais bouillis consommés au Bénin
}

\author{
Franck HONGBETE ${ }^{1,2}$, Janvier M. KINDOSSI ${ }^{1,2^{*}}$, Joseph D. HOUNHOUIGAN ${ }^{2}$ et \\ Mathurin C. NAGO ${ }^{2}$
}

${ }^{I}$ Département de Nutrition et Sciences Agro-Alimentaires, Faculté d'Agronomie, Université de Parakou, BP : 123 Parakou, Bénin.

${ }^{2}$ Laboratoire de Biochimie Microbienne et Biotechnologie Alimentaire,

Faculté des Sciences Agronomiques, Université d'Abomey-Calavi, 01 B.P. 526 Cotonou, Bénin.

${ }^{*}$ Auteur correspondant ; E-mail : jkindossi@gmail.com,Tel : 0022996814420

\section{RESUME}

L'épi de maïs frais bouilli est l'un des aliments obtenus de la transformation du maïs. L'objectif de l'étude est d'évaluer les facteurs socio-économiques des acteurs, de caractériser la technologie de production et de déterminer la qualité nutritionnelle du produit. A cet effet, une enquête semi-structurée a été réalisée à Cotonou et Parakou, deux villes cosmopolites du Bénin. Parallèlement, les échantillons collectés ont été soumis aux analyses physicochimiques. Les résultats ont montré que la production des épis de maïs frais bouillis est une activité féminine prédominante en période de disponibilité. La technologie de préparation des épis de maïs frais bouillis diffère d'une région à une autre. Ainsi, à Parakou, l'épi de maïs frais est bouilli avec les spathes alors qu'il est bouilli sans les spathes à Cotonou. Selon les consommateurs, la date post-récolte affecte le goût et la texture des maïs bouillis. Pour les transformatrices-vendeuses et les consommateurs, un bon maïs frais bouilli doit avoir une texture moins dure et un goût succulent. Les caractéristiques nutritionnelles du maïs frais bouilli révèlent une richesse en glucides totaux $(63,4$ à $64,6 \%$ matière sèche), en protéines $(12,5 \%$ matière sèche), en minéraux ( $1,6 \%$ matière sèche) et composés phénoliques ( 5,5 à $6,1 \%$ matière sèche).

(C) 2017 International Formulae Group. All rights reserved.

Mots clés : Maïs, valeur nutritionnelle, appréciation, consommateurs, Bénin.

\section{Production and nutritional quality of cob of boiled fresh maize consumed in Benin}

\begin{abstract}
Cob of boiled fresh maize is one of foods obtained from maize processing. The aim of the study was to evaluate the socio-economic factors of the stakeholders, to characterize the production technology and to determine the nutritional quality of the product. A semi-structured survey was carried out in Cotonou and Parakou, two cosmopolitan cities of Benin. On the other hand, the collected samples have been subjected to physicochemical analysis. The results have shown that the production of cob of boiled fresh maize is a prevalent female activity in its available period. The technology of production of cob of boiled fresh maize differs from one area to another. Thus, in Parakou, the cob of fresh maize is boiled with the spathes whereas it
\end{abstract}


is boiled without the spathes in Cotonou. According to consumers, the post-harvest date affects the taste and texture of boiled maize. For the processor-sellers and consumers, a good boiled fresh maize must have a less hard texture and luscious taste. The nutritional characteristics of boiled maize reveal a high total carbohydrate (63.4 to $64.6 \%$ dry matter), protein $(12.5 \%$ dry matter), mineral (1.6\% dry matter) and phenolic compounds (5.5 to $6.1 \%$ dry matter).

(C) 2017 International Formulae Group. All rights reserved.

Keywords: Maize, nutritional value, appreciation, consumers, Benin.

\section{INTRODUCTION}

Le maïs (Zea mays) est une céréale de grande importance alimentaire pour de nombreuses populations en Afrique de l'Ouest, principalement au Bénin où il constitue la base du régime alimentaire des populations du Sud et du Centre du Pays (Adégbola et al., 2011; Sarr et al., 2011). Bien que le maïs soit longtemps considéré comme un produit de subsistance et de soudure, sa culture s'est aujourd'hui intensifiée et répandue dans toutes les régions du Bénin en raison des avantages nutritionnels (richesse en amidon, présence de protéines, bonne matière grasse) et économiques (culture facile et peu coûteuse) qu'il offre (Adégbola et al., 2011). La production nationale de maïs a été estimée en 2012 à 1175000 tonnes au Bénin (FAO, 2013). Le niveau moyen de consommation de maïs est estimé à plus de 85 $\mathrm{kg} /$ habitant/an, ce qui place le pays au premier rang des pays grands consommateurs en Afrique de l'Ouest (Adégbola et al., 2011 ; Abadassi, 2014). Compte tenu de l'importance que revêt le maïs pour la sécurité alimentaire au Bénin, cette céréale a été retenue comme filière prioritaire pour la relance du secteur agricole dans le Plan Stratégique de Relance du Secteur Agricole du Bénin (PSRSA) par le Ministère de l'agriculture de l'Elevage et de la Pêche (MAEP) (PSRSA, 2010). Ainsi sa promotion serait intéressante et nécessaire pour assurer la sécurité alimentaire et la croissance économique du Bénin (Baco et al., 2010). Le maïs est utilisé en alimentation humaine sous diverses formes selon les régions et les habitudes alimentaires des groupes socio-culturels (Nago, 1997 ; Adégbola et al., 2011 ; Houngbo, 2015). En plus de son utilisation dans l'alimentation humaine et animale, le maïs sert aussi de matière première dans certaines industries (Houngbo, 2015 ; Ba et al., 2016). Les principaux produits dérivés de sa transformation sont le maïs frais bouilli ou grillé, les farines ou semoule de maïs, la pâte, l'akassa, le mawè et la bouillie (Nago, 1997 ; Semassa et al., 2016). Le maïs frais bouilli constitue l'une des principales formes d'utilisation du produit. La quantité de maïs frais incluse dans le secteur de préparation du maïs bouilli est importante mais aucune statistique sur cette quantité n'est encore disponible au Bénin. La préparation et la commercialisation du maïs frais est une activité génératrice de revenus qui est pratiquée aussi bien dans les zones urbaines que dans les zones rurales du Bénin (Baco et al., 2010). Différentes techniques de cuisson sont pratiquées dans la préparation du maïs frais dans ces zones. D'après les consommateurs, la qualité du maïs frais varie énormément. La période post-récolte et la variété sont souvent soupçonnées comme les facteurs affectant cette qualité. Les études antérieures faites sur le maïs au Bénin se sont surtout focalisées sur les aspects liés à la production et à la commercialisation du produit (Baco et al., 2010) ainsi que la caractérisation microbiologique et physicochimique du mawè et du ogui, deux pâtes fermentées à base de maïs (Nago, 1997; Semassa et al., 2016). Les caractéristiques de la qualité du maïs frais bouilli ne sont pas encore décrites. La préparation et la commercialisation du maïs frais bouilli étant une activité qui s'intensifie au Bénin et dans la sous-région Ouest africaine, il serait 
intéressant d'investiguer sur les caractéristiques socio-économiques des acteurs et la qualité nutritionnelle du produit. Cette étude vise donc à évaluer les caractéristiques socio-économiques des acteurs, les techniques de préparation utilisées, les critères d'appréciation de la qualité et à déterminer les caractéristiques nutritionnelles du maïs frais bouilli.

\section{MATERIEL ET METHODES \\ Zone d'étude}

L'étude s'est déroulée du 12 août au 29 octobre 2015 dans la commune de Cotonou située entre $2^{\circ} 20^{\prime}$ et $2^{\circ} 29^{\prime}$ longitude Est puis $6^{\circ} 20^{\prime}$ et $6^{\circ} 25^{\prime}$ latitude Nord et dans la commune de Parakou localisée entre $2^{\circ} 23^{\prime}$ et $2^{\circ} 44^{\prime}$ longitude Est puis entre $9^{\circ} 14^{\prime}$ et $9^{\circ} 29^{\prime}$ latitude Nord (Figure 1). Ces deux communes ont été choisies compte tenu de leur caractère cosmopolite, de leur forte densité en population et de la diversité culturelle des peuples au plan social et alimentaire.

\section{Enquête}

Une pré-enquête a permis de tester un questionnaire préétabli auprès de cinq (05) transformatrices-vendeuses de l'épi de maïs frais bouilli et vingt (20) consommateurs parlant différentes langues locales. Cette technique a permis d'adapter le questionnaire aux réalités sociologiques. Les diverses langues parlées étaient le Dendi, le Bariba, le Fon et le Mina. Ensuite, le questionnaire a été adressé de façon individuelle aux transformatrices-vendeuses et aux consommateurs de maïs frais bouilli.

La taille de l'échantillon a été déterminée selon la méthode de boule de neige (Hongbété et Kindossi, 2017). Cette méthode a consisté à un ratissage systématique des transformatrices-vendeuses de maïs frais bouilli. $\mathrm{Au}$ total, 120 transformatrices-vendeuses de maïs bouilli dont 45 à Parakou et 75 à Cotonou ont été enquêtées dans les localités ciblées. Alors que 241 consommateurs de maïs frais bouilli à savoir 90 à Parakou et 151 à Cotonou ont été enquêtés soit deux (02) consommateurs par transformatrice-vendeuse.

Les informations mentionnées sur le questionnaire servant de guide d'entretien sont relatives au statut sociodémographique de l'enquêté (identité, domaine d'activités, genre, niveau de scolarisation, âge, ethnie), aux aspects technologiques du traitement du maïs frais et les préférences des consommateurs selon la période post-récolte et la qualité organoleptique (texture, goût et couleur) du maïs.

\section{Analyses physicochimiques}

Des échantillons de deux variétés de maïs (blanche et jaune) collectés dans les zones d'enquête ont été analysés. Ainsi, la matière sèche a été déterminée par séchage de $5 \mathrm{~g}$ d'échantillon de grains de maïs à l'étuve à $105^{\circ} \mathrm{C}$, jusqu'à obtention de masse constante. $\mathrm{La}$ teneur en matière minérale a été déterminée suivant la méthode AOAC (1995) après incinération au four à $550{ }^{\circ} \mathrm{C}$ pendant 24 heures de $5 \mathrm{~g}$ d'échantillon de maïs frais broyé. Les taux de matière grasse ont été déterminés à partir de la matière sèche de la farine de maïs selon la méthode AOAC (1995) utilisant le SOXHLET. Les glucides totaux ont été dosés selon la méthode décrite par Tshite et al. (2015). Quant aux protéines, leurs teneurs ont été déterminées selon la méthode AOAC (1995). La méthode de Singleton et al. (1999) a été utilisée pour doser les phénols totaux.
Analyses statistiques
Les données de l'enquête ont été dépouillées, organisées et traitées grâce au logiciel Sphinx plus ${ }^{2}$ version 5.1.0.4. Les résultats d'analyses physicochimiques ont été soumis à une analyse de variance (ANOVA) à un facteur suivi du test de Newman-keuls grâce au logiciel Statistica version 7.1 StatSoft France (2006). La significativité de la différence entre les échantillons de maïs a été déterminée au seuil de $5 \%$. 


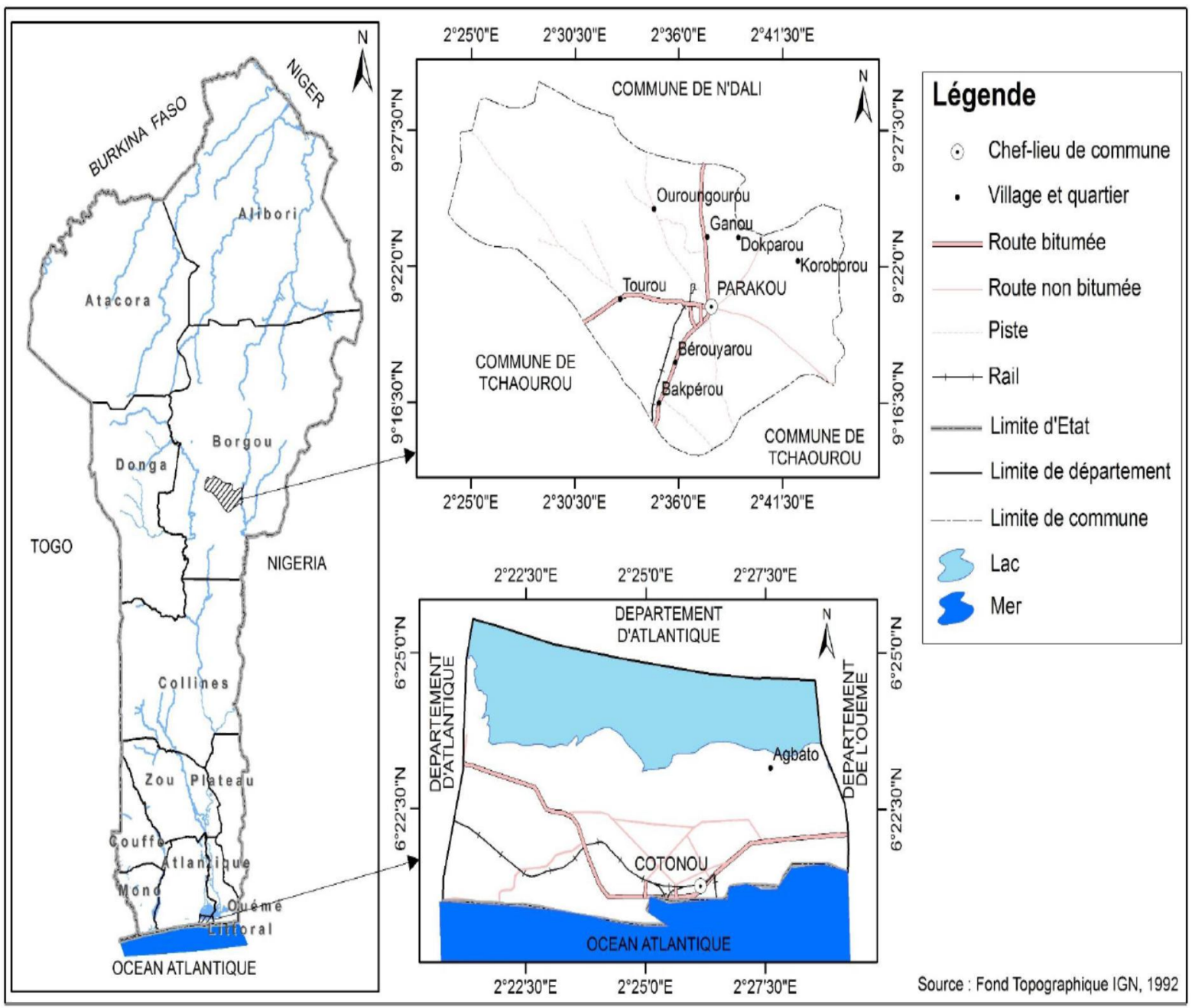

Figure 1 : Localisation géographique des zones d'étude au Bénin.

\section{RESULTATS}

Caractéristiques socio-culturelles des acteurs liés à la transformation-vente et la consommation du maïs frais bouilli au Bénin

Le Tableau 1 présente les caractéristiques socioculturelles des transformatrices-vendeuses et consommateurs de maïs frais bouillis. Ainsi, la transformation-vente des épis de maïs frais bouillis est une activité essentiellement féminine (100\% des enquêtées) qui occupe une population de femmes majoritairement analphabète ( $70 \%$ des enquêtées). Seulement $30 \%$ des femmes impliquées dans ce secteur d'activité ont fait les études primaires. Cette population est répartie en quatre tranches d'âges dont la première (âgées de 18 à 25 ans) représente $33,33 \%$ des enquêtées, la deuxième (26 à 35 ans) représente 37,50\%; la troisième (36 à 45 ans) représente $25 \%$, et la dernière tranche de transformatrices-vendeuses (46 à 
50 ans) relativement passives, représente $4,16 \%$ des enquêtées. Ces transformatricesvendeuses appartiennent à 6 groupes socioethniques constitués majoritairement des Fon $(38,33 \%)$ et des Aïzo (37,5\%). La plupart (96,5\%) des transformatrices-vendeuses ont déclaré que cette activité ne nécessite pas une expérience dans le domaine.

Concernant les consommateurs, l'épi de maïs frais bouilli est consommé majoritairement par les groupes socioculturels Aïzo (21,67\%), Mina (15\%), Adja (11,67\%), Fon $(10,83 \%)$, Dendi $(10,42 \%)$, Goun (10\%), Nagot $(8,33 \%)$. Les autres groupes rencontrés minoritairement sont les Yoruba, Bariba et Wama qui représentent $6,25 \%, 4,17 \%$ et $1,67 \%$ des enquêtés, respectivement. La majorité des consommateurs de maïs frais bouilli sont constitués d'universitaires $(36,93 \%)$ et de collégiens $(23,65 \%)$. Les autres n'ont pas terminé les études primaires $(17,84 \%)$, et le reste $(21,58 \%)$ n'est pas allé à l'école.

\section{Transformation du maïs frais et critère d'appréciation des variétés utilisées}

La transformation et la vente de l'épi de maïs frais est une activité saisonnière qui s'effectue essentiellement de mars à août et de mi-octobre à fin novembre au Sud Bénin et de juin à novembre au Nord Bénin. Toutefois, il existe des productions agricoles de maïs de contre saison.

Deux principales variétés de maïs, à savoir la variété blanche et la variété jaune, sont couramment utilisées pour la préparation de l'épi de maïs frais bouilli au Bénin. Les transformatrices-vendeuses s'en approvisionnent aux abords des champs, au niveau des points de regroupement dans les marchés locaux. Selon ces transformatricesvendeuses, le premier critère de qualité utilisé dans le choix de la matière première est la couleur des grains. Ainsi, le maïs jaune est plus apprécié $(65,83 \%$ des enquêtés) que le maïs blanc $(34,17 \%)$ (Tableau 2). Ce choix est lié au goût relativement sucré et à la succulence caractéristique du maïs jaune. D'après les transformatrices-vendeuses, les consommateurs préfèrent le maïs jaune au maïs blanc. Pour ces consommateurs, le maïs jaune a un goût plus sucré et plus succulent que le maïs blanc. Le goût du maïs est très important dans le choix de ce produit. Il dépend de plusieurs facteurs dont la variété et la durée post-récolte précédant la cuisson. Le second critère évoqué par les transformatricesvendeuses est relatif à l'aspect des spathes. En effet, les spathes doivent être intacts $(40 \%$ des enquêtés), c'est-à-dire dépourvu de larve et de charançon. De plus, les spathes doivent être de couleur verte ( $58 \%$ des enquêtés) avec un goût sucré au niveau de la rafle (30\% des enquêtés) (Figure 2). La couleur des spathes est un indicateur de la fraîcheur et du goût du maïs. Ainsi, plus les spathes de maïs sont fraîches (couleur verte vive), plus le goût sucré du maïs est intense, ce qui indique que le maïs est fraîchement récolté. En revanche, la couleur moins verte des spathes de maïs indique un goût moins sucré et une longue période postrécolte.

\section{Quantité et fréquences de préparation des épis de maïs frais}

Le Tableau 3 présente les quantités et fréquences de préparation des épis de maïs frais. La quantité d'épis de maïs frais préparés par semaine varie selon la catégorie de transformatrices-vendeuses entre 200 et 800 $\mathrm{kg}$. En effet, la majorité des transformatricesvendeuses d'épis de maïs frais $(53,6 \%$ pour le maïs blanc et $82,2 \%$ pour le mais jaune) transforment en moyenne entre 200 et $300 \mathrm{~kg}$ 
de maïs frais par semaine. Environ 7,3\% des transformatrices-vendeuses de maïs blanc transforment jusqu'à $800 \mathrm{~kg}$ de maïs frais alors que $12,7 \%$ des transformatricesvendeuses de maïs jaune préparent entre 300 et $450 \mathrm{~kg}$ de maïs frais par semaine.

En ce qui concerne la fréquence de préparation, elle tourne autour de 3 à 5 fois par semaine $(63,4 \%$ des transformatricesvendeuses de maïs blanc et $75,9 \%$ des transformatrices-vendeuses de maïs jaune). $\mathrm{Au}$ cours de la période d'abondance, seulement quelques transformatricesvendeuses de maïs blanc $(9,8 \%)$ et de maïs jaune $(6,3 \%)$ préparent les épis de maïs frais presque tous les jours de la semaine alors que pendant la période de rareté, la fréquence de production est 2 fois par semaine.

\section{Modes de préparation du maïs frais et attributs de qualité du produit fini}

Pour la production des épis de maïs frais bouillis, deux modes de préparation ont été recensés dans les régions étudiées. Le premier est le plus répandu à Cotonou $(62,5 \%$ des enquêtés). Il consiste à préparer les épis de maïs débarrassés de leurs spathes et des fils en soie. Pour le second mode de préparation généralement en usage à Parakou $(77,5 \%$ des enquêtés), les épis ne sont pas dépourvus de leurs spathes avant la cuisson mais les fils de soie sont juste coupés à l'aide d'une machette. Ensuite, les épis déspathés ou non sont lavés pour éliminer les impuretés puis disposés dans une marmite, recouverts d'eau et bouillis pendant environ 1 heure (Figure 3).

Concernant la qualité du maïs frais bouilli, les principaux attributs de qualité utilisés par les acteurs sont indiqués dans le Tableau 4. Environ 39,2 à $52,5 \%$ des transformatrices-vendeuses des épis de maïs frais ont cité la couleur comme un des principaux attributs de qualité du maïs frais. Ceci est confirmé par 40,2 à $82,5 \%$ des consommateurs d'épis de maïs frais bouillis. La texture constitue également un important attribut de qualité de l'épi de maïs frais bouilli. Plus de la moitié $(53,7 \%)$ des transformatrices-vendeuses et la majorité $(89,8 \%)$ de consommateurs enquêtés estiment que la texture des épis de maïs frais bouillis doit être moins dure. Concernant le goût, la majorité des transformatrices-vendeuses $(74,8 \%)$ et des consommateurs $(81,6 \%)$ sont tous d'accord que l'épi de maïs frais bouilli doit être succulent et relativement sucré.

En ce qui concerne le goût salé, 70,3\% des transformatrices-vendeuses ont recours au salage pour camoufler le goût fade caractéristique des maïs frais bouillis 24 et 48 heures après récolte.

Par rapport à l'odeur, les transformatrices-vendeuses interviewées (78,9\%) ont déclaré que l'épi de maïs frais bouilli doit avoir l'odeur caractéristique du maïs frais. Ceci paraît un important critère de qualité comme l'ont soutenu la majorité $(97,1 \%)$ des consommateurs.

\section{Facteurs influençant le prix de vente du maïs frais bouilli}

Selon $81,2 \%$ des enquêtés, le prix d'achat de la matière première est un facteur déterminant du prix de vente des épis de maïs frais bouillis. Ce prix varie d'une localité à une autre suivant les saisons de récolte. Il dépend également des coûts de transport de la matière première liés à l'éloignement des zones d'approvisionnement. Ainsi, les épis de maïs frais bouillis sont plus chers en période de contre saison, en début et en fin de saison. Il est également plus cher en milieu urbain qu'en zone rurale. 
Lieu et moment de consommation des épis de maïs frais bouillis

L'épi de maïs frais bouilli est un aliment de rue. $\mathrm{Vu}$ comme tel, les consommateurs sont rencontrés dans les lieux publics, la rue $(90,8 \%)$, à la maison $(28,3 \%)$ et sur leur lieu de travail (2,2\%). La majorité des enquêtés $(81,7 \%)$ consomment l'épi de maïs frais bouilli au déjeuner (60\%), entre les repas $(34 \%)$ et au dîner (6\%) (Figure 4). Généralement, l'épi de maïs frais bouilli est consommé avec du coprah (34\%) ou de l'arachide bouillie ou grillée (58\%).

Préférence de maïs frais bouilli suivant la période post-récolte par classes de population

L'épi de maïs frais bouilli est consommé par toutes les classes d'âges de la société béninoise parmi lesquelles s'y trouvent des adolescents, des personnes non vulnérables, des personnes âgées, des personnes malades, des femmes enceintes et des femmes allaitantes (Figure 5). Toutefois, d'après les enquêtes, les personnes malades $(6,53 \%)$ et les personnes âgées $(5,31 \%)$ consomment moins d'épi de maïs frais bouilli et n'aiment pas du tout les épis bouillis 48 heures après récolte (MB48AR). Elles ont déclaré que la texture de l'épi de maïs bouilli 48 heures après récolte est plus dure que celle de l'épi bouilli le jour de récolte (MBJR). De plus, l'épi de maïs bouilli présentant une texture dure est difficile à mastiquer et fatigue plus les mâchoires. Les adolescents (26,46\%) sont indifférents car ils consomment l'épi de maïs bouilli quelle que soit la date post-récolte alors que les personnes non vulnérables $(18,29 \%)$, les femmes enceintes $(21,42 \%)$ et les femmes allaitantes $(22,57 \%)$ consomment plus l'épi de maïs bouilli le jour de récolte (MBJR) que ceux bouillis 24 heures
(MB24AR) ou 48 heures après récolte (MB48AR). Ces classes ont évoqué que l'épi de maïs frais bouilli le jour de récolte est plus succulent que celui bouilli 24 heures ou 48 heures après récolte. Aussi, environ 25\% des femmes enceintes enquêtées aiment l'épi de maïs bouilli 48 heures après récolte. Ceci est rattaché à la dureté des grains qui leur permet d'avoir à grignoter pendant un long moment.

\section{Caractéristiques nutritionnelles des variétés de maïs frais bouilli}

Le Tableau 5 présente les caractéristiques nutritionnelles de deux variétés de maïs frais bouilli collectées dans les différentes zones d'enquête. La teneur en eau de la variété jaune (20,9\%) est significativement supérieure au seuil de $5 \%$ à celle de la variété blanche $(20,2 \%)$. La teneur en matière grasse de la variété jaune $(3,4 \%$ MS) est significativement plus élevée au seuil de $5 \%$ que celle de la variété blanche $(2,8 \%$ MS). Cette comparaison montre que le maïs jaune contient plus de lipides que le maïs blanc. Aucune différence significative n'a été observée entre la teneur en glucides totaux de la variété jaune $(64,6 \% \mathrm{MS})$ et celle de la variété blanche $(63,4 \%)$. Les teneurs en protéines des variétés de maïs sont comprises entre 12,4 et $12,6 \%$ MS et leurs teneurs en cendres varient de 1,6 à $1,7 \%$. La comparaison des moyennes des teneurs en constituants des échantillons montre qu'il n'y a pas de différence significative entre les teneurs en protéines et en cendres des deux variétés de maïs étudiées. La teneur en composés phénoliques de la variété jaune (6,1\% MS) est significativement supérieure au seuil de $5 \%$ à celle de la variété blanche $(5,5 \%$ MS) 
Tableau 1: Caractéristiques socioculturelles des transformatrices-vendeuses et consommateurs enquêtés.

\begin{tabular}{|c|c|c|c|c|c|}
\hline \multirow[b]{2}{*}{ Variables } & & \multicolumn{2}{|c|}{$\begin{array}{c}\begin{array}{c}\text { Transformatrices- } \\
\text { vendeuses }\end{array} \\
\end{array}$} & \multicolumn{2}{|l|}{ Consommateurs } \\
\hline & & $\begin{array}{c}\text { Nombre } \\
\text { ayant cité } \\
(\mathrm{n}=120)\end{array}$ & $\begin{array}{c}\text { Fréquence } \\
(\%)\end{array}$ & $\begin{array}{l}\text { Nombre ayant } \\
\text { cité }(n=241)\end{array}$ & $\begin{array}{c}\text { Fréquence } \\
(\%)\end{array}$ \\
\hline \multirow{6}{*}{ Ages (ans) } & $\leq 17$ & - & - & 20 & 8,3 \\
\hline & $18-25$ & 40 & 33,33 & 41 & 17,01 \\
\hline & $26-35$ & 45 & 37,5 & 40 & 16,6 \\
\hline & $36-45$ & 30 & 25 & 50 & 20,75 \\
\hline & $46-50$ & 5 & 4,16 & 55 & 22,82 \\
\hline & $\geq 51$ & & & 35 & 14,52 \\
\hline \multirow{2}{*}{ Sexe } & Femmes & 120 & 100 & 138 & 57,26 \\
\hline & Hommes & - & - & 103 & 42,74 \\
\hline \multirow{4}{*}{$\begin{array}{l}\text { Niveau } \\
\text { d'étude }\end{array}$} & N'est pas allé à l'école & 98 & 70 & 52 & 21,58 \\
\hline & Primaire & 42 & 30 & 43 & 17,84 \\
\hline & Secondaire & - & - & 57 & 23,65 \\
\hline & Universitaire & - & - & 89 & 36,93 \\
\hline \multirow{10}{*}{$\begin{array}{l}\text { Groupe } \\
\text { socio } \\
\text { culturel }\end{array}$} & Dendi & 2 & 1,67 & 25 & 10,42 \\
\hline & Nagot & 5 & 4,17 & 20 & 8,33 \\
\hline & Fon & 46 & 38,33 & 27 & 10,83 \\
\hline & Aïzo & 45 & 37,5 & 52 & 21,67 \\
\hline & Goun & 12 & 10 & 24 & 10 \\
\hline & Adja & 10 & 8,33 & 28 & 11,67 \\
\hline & Mina & - & - & 36 & 15 \\
\hline & Yoruba & - & - & 15 & 6,25 \\
\hline & Bariba & - & - & 10 & 4,17 \\
\hline & Wama & - & - & 4 & 1,67 \\
\hline
\end{tabular}

$\mathrm{n}$ : nombre de personnes enquêtés.

Tableau 2 : Fréquence de distribution du choix de la variété de maïs par les transformatricesvendeuses.

\begin{tabular}{lccc}
\hline Matière première & Variété & $\begin{array}{c}\text { Nombre ayant cité } \\
(\mathbf{N = 1 2 0})\end{array}$ & Fréquence (\%) \\
\hline \multirow{2}{*}{ Maïs } & Blanche & 41 & 34,17 \\
& Jaune & 79 & 65,83 \\
\hline
\end{tabular}




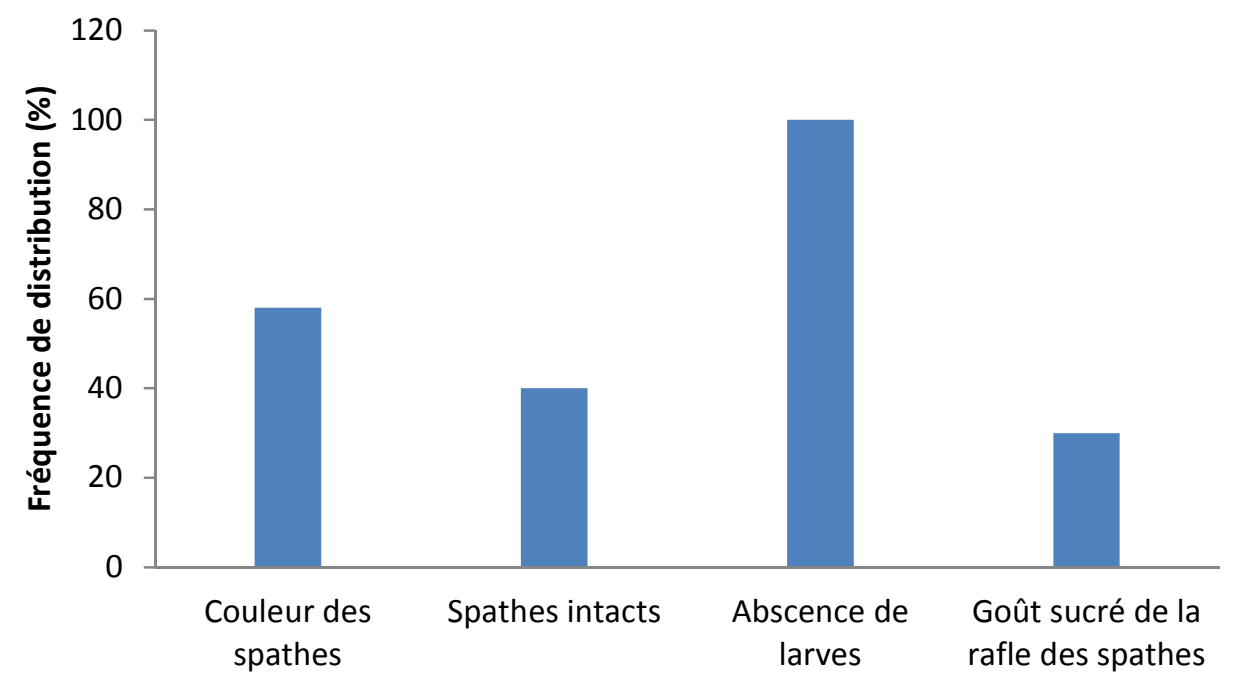

Figure 2 : Fréquence de distribution des enquêtés ayant évoqué une qualité requise de la matière première.

Tableau 3 : Quantité de maïs frais et fréquence de préparation des épis de maïs bouillis.

\begin{tabular}{lcc}
\hline & \multicolumn{2}{c}{ Transformatrices-vendeuses } \\
\hline & $\begin{array}{c}\text { Maïs blanc } \\
\mathbf{n}=\mathbf{4 1}\end{array}$ & $\begin{array}{c}\text { Maïs jaune } \\
\mathbf{n}=\mathbf{7 9}\end{array}$ \\
& $\mathbf{( \% )}$ & $\mathbf{( \% )}$ \\
\hline Quantité de maïs frais (kg/semaine) & & \\
{$[200-300[$} & 53,6 & 82,2 \\
{$[300-450[$} & 24,4 & 12,7 \\
{$[450-600[$} & 14,6 & - \\
{$[600-800[$} & 7,3 & - \\
\hline Fréquence (par semaine) & & \\
1-2 & 26,8 & 17,7 \\
$3-5$ & 63,4 & 75,9 \\
$6-7$ & 9,8 & 6,3 \\
\hline
\end{tabular}




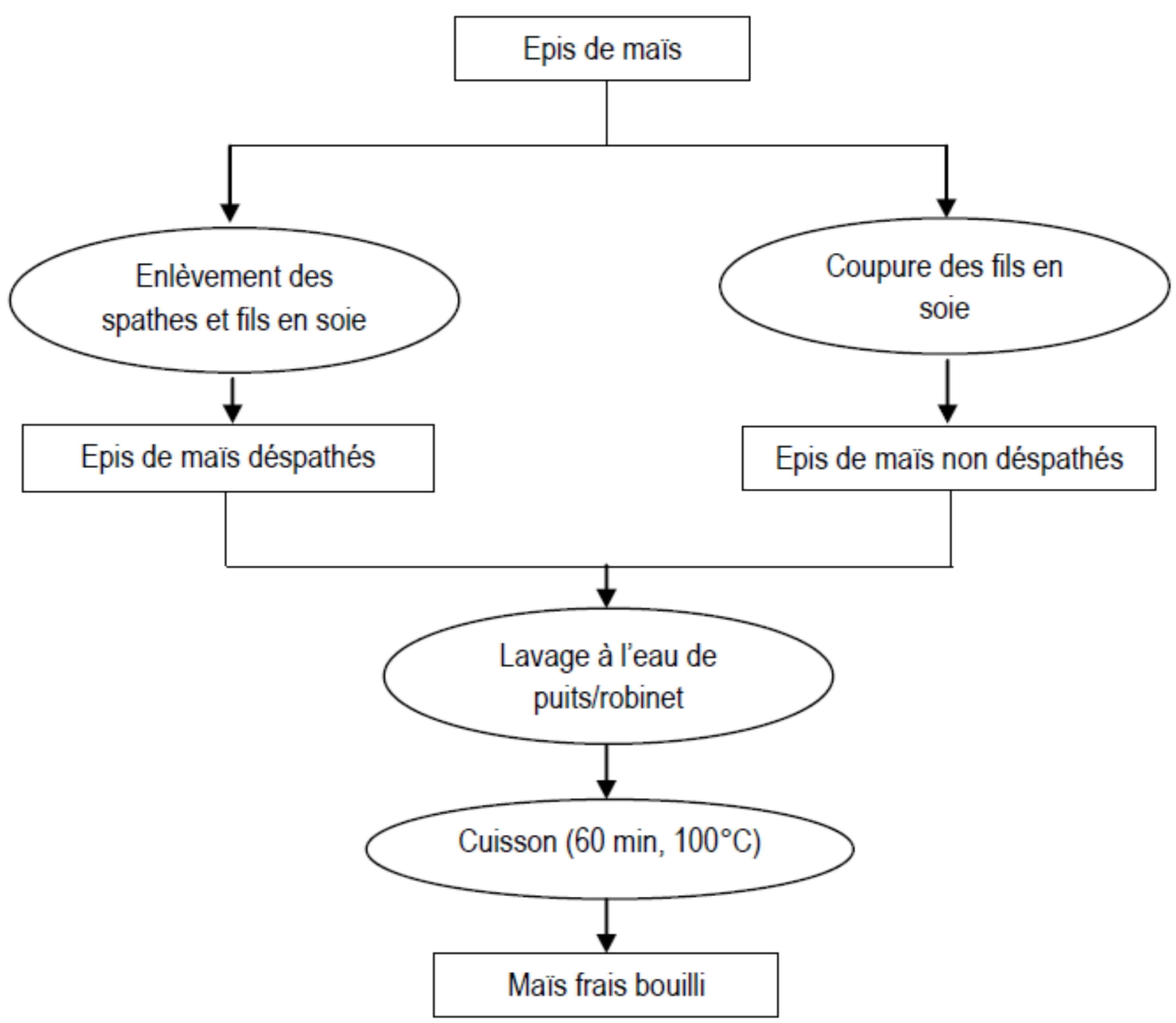

Figure 3 : Diagramme de préparation des épis de maïs frais bouillis.

Tableau 4 : Attribut de qualité des épis de maïs frais bouillis et leur importance (\% des enquêtés) selon les acteurs.

\begin{tabular}{lcc}
\hline \multirow{2}{*}{ Attributs sensoriels } & Transformatrices vendeuses & Consommateurs \\
\cline { 2 - 3 } & Maïs frais bouilli & $\begin{array}{l}\text { Maïs } \\
\text { bouilli }\end{array}$ \\
\hline Couleur blanche & 39,2 & 40,2 \\
Couleur jaune & 52,5 & 82,5 \\
Texture moins dure & 53,7 & 89,8 \\
Succulent & 74,8 & 81,6 \\
Goût sucré & 24,7 & 59,3 \\
Goût salé & 70,3 & 45,4 \\
Goût fade & 3,2 & 1,4 \\
Odeur de maïs & 78,9 & 97,1 \\
\hline
\end{tabular}




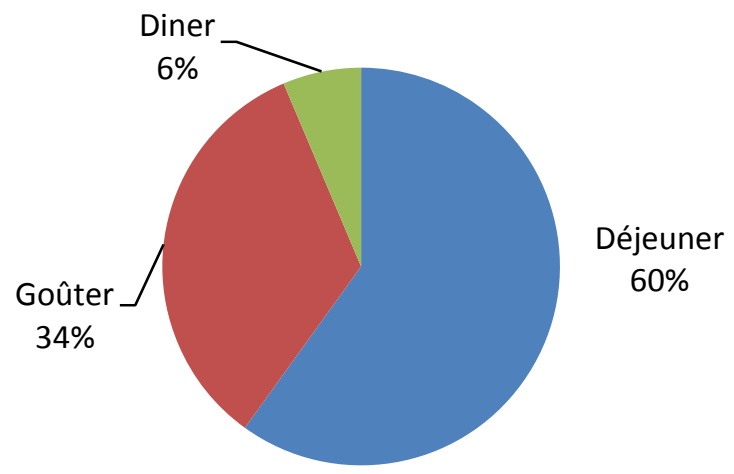

Figure 4 : Moment de consommation des épis de maïs frais bouillis.

Tableau 5 : Caractéristiques nutritionnelles des maïs frais bouillis.

\begin{tabular}{lcc}
\hline Paramètres & \multicolumn{2}{c}{ Maïs } \\
\cline { 2 - 3 } & Blanc $(\mathbf{n}=\mathbf{6})$ & Jaune $(\mathbf{n}=\mathbf{6})$ \\
\hline Teneur en eau (\%) & $20,2 \pm 0,2^{\mathrm{a}}$ & $20,9 \pm 0,4^{\mathrm{b}}$ \\
Matière grasse (\%MS) & $2,8 \pm 0,0^{\mathrm{a}}$ & $3,4 \pm 0,5^{\mathrm{b}}$ \\
Glucides totaux (\%MS) & $63,4 \pm 0,4^{\mathrm{a}}$ & $64,6 \pm 0,9^{\mathrm{a}}$ \\
Protéines (\%MS) & $12,4 \pm 0,1^{\mathrm{a}}$ & $12,6 \pm 0,1^{\mathrm{a}}$ \\
Teneur en cendres (\%MS) & $1,6 \pm 0,5^{\mathrm{a}}$ & $1,7 \pm 0,4^{\mathrm{a}}$ \\
Composés Phénoliques (\%MS) & $5,5 \pm 0,1^{\mathrm{a}}$ & $6,1 \pm 0,2^{\mathrm{b}}$ \\
\hline
\end{tabular}

$\overline{a, b}$ valeurs affectées avec les mêmes lettres sur une même ligne ne sont pas significativement différentes au seuil de 5\% (Test de Newman-keuls). $\mathrm{n}$ : le nombre d'échantillons analysés; MS : matière sèche

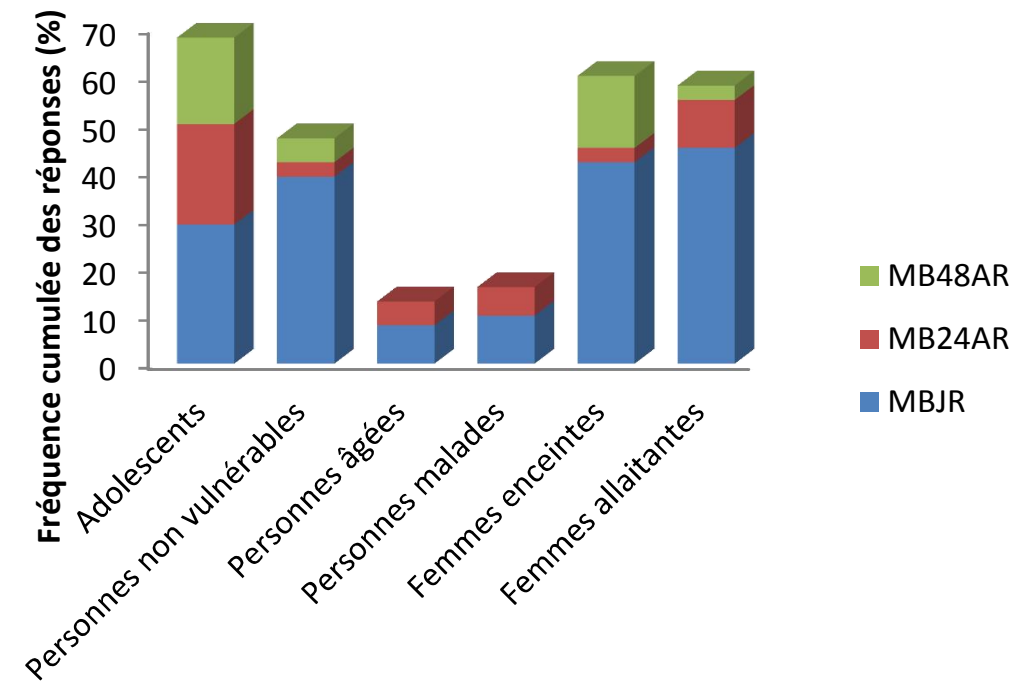

Figure 5 : Préférence de consommation de l'épi de maïs frais bouilli suivant la période post-récolte selon la classe de la population. MBJR : épi de maïs bouilli le jour de la récolte; MB24AR : épi de maïs bouilli 24 heures après récolte; MB48AR : épi de maïs bouilli 48 heures après récolte. 


\section{DISCUSSION}

Le maïs (Zea mays L.) constitue l'alimentation de base pour de nombreuses populations à qui il procure l'essentiel de l'énergie nécessaire (Sarr et al., 2011). Il est transformé grâce à l'artisanat en une diversité de produits alimentaires dont l'épi de maïs frais bouilli (Sodjinou et al., 2008 ; Semassa et al., 2016). L'étude des caractéristiques socioéconomiques des acteurs a montré que la production de l'épi de maïs frais bouilli est une activité essentiellement féminine qui occupe des femmes de différentes tranches d'âge avec un faible niveau d'instruction. Ce constat a été également souligné par d'autres auteurs qui ont remarqué que les activités de production et de commercialisation des produits alimentaires de rue sont généralement assurées par les femmes majoritairement peu instruites (Kayodé et al., 2006 ; Kindossi et al., 2012 ; Sacca et al., 2012 ; Hongbété et al., 2017).

De plus, l'étude a révélé que l'épi de maïs frais bouilli est un aliment préféré des béninois. Il est consommé par toutes les couches sociales, quelles que soient leur niveau d'éducation et leur situation économique. Cet intérêt des consommateurs pour ce produit confirme l'importance du maïs dans le fonctionnement du système alimentaire du Bénin (Nago, 1997 ; Abadassi, 2014 ; Houngbo, 2015 ; Semassa et al., 2016).

La technologie de transformation de l'épi de maïs frais est traditionnelle et varie d'une région à une autre. Les variétés de maïs utilisées pour cette transformation sont principalement la variété blanche et la variété jaune. Ces deux variétés ont été également évoquées dans une étude antérieure concernant la caractérisation de la production traditionnelle des cultures de maïs au Bénin (Abadassi, 2014).

Aussi, d'autres auteurs (Nago, 1997 ; Mestres et al., 1997 ; Semassa et al., 2016) ont indiqué, par ailleurs, que les caractéristiques des variétés de maïs conditionnent leur comportement au cours de la transformation mécanique. Avec l'urbanisation, l'exode rural et l'intensification du phénomène d'alimentation de rue, la production de l'épi de maïs frais bouilli a pris une ampleur considérable au Bénin. En effet, selon Nago
(1997), les produits de maïs sont très appréciés en milieu urbain surtout pendant les périodes de disponibilité. Selon les transformatrices-vendeuses et les consommateurs, l'épi de maïs frais bouilli doit avoir une texture moins dure, un goût succulent et une odeur caractéristique de maïs frais.

Le goût de l'épi de maïs frais bouilli est fonction du moment de la cuisson après récolte. Plus la période post-récolte précédant la cuisson de l'épi de maïs frais est courte, plus le produit est succulent et mieux apprécié par les consommateurs. Autrement, les longues périodes post-récoltes (épi de maïs frais bouilli 24 ou 48 heures après récolte) ont des influences négatives sur l'aspect des graines du maïs frais, le goût, la texture et l'arôme dégagé par le maïs lors de sa préparation. Ces qualités sont le plus souvent reconnues sur le maïs à l'état frais par la douceur de la rafle des spathes et des grains.

De plus, concernant les teneurs en eau et en matière grasse, les résultats obtenus ont montré des différences entre variétés. De même, Deffan et al. (2015) ont rapporté, dans une étude d'évaluation morphologique et nutritionnelle de variétés locales et améliorées de maïs produites en Côte d'Ivoire, des différences sur ces paramètres physicochimiques entre variétés.

Les épis de maïs frais bouillis ont une humidité du grain supérieure à $20 \%$. Une telle teneur en eau du maïs bouilli augmente le risque de détérioration et de croissance microbienne du produit s'il n'est pas immédiatement consommé. $\mathrm{Ce}$ taux d'humidité des grains de maïs frais bouillis sont supérieurs à ceux déterminés par Enyisi et al. (2014) qui ont trouvé des taux d'humidité variant entre 11 et $20 \%$ dans les grains de maïs. Cette différence peut être attribuée à la variété de maïs utilisée, aux facteurs environnementaux et aux pratiques agronomiques.

Les maïs frais bouillis sont très riches en glucides. Les glucides représentent la portion la plus importante de la matière sèche des céréales. Le dosage des glucides est d'apprécier la valeur énergétique des variétés de maïs étudiées. Aussi, la présence de la matière grasse, de protéines en proportion 
intéressante montre l'importance nutritionnelle des variétés de maïs étudiées. Les différences observées sont spécifiquement dues au type de variété. Ainsi, la variété jaune étudiée présente une qualité nutritionnelle plus intéressante que la variété blanche. Les teneurs en glucides totaux $(63,4$ à $64,6 \% \mathrm{MS})$ se rapprochent de celles $(59,4$ et $77,7 \% \mathrm{MS})$ des variétés de maïs locales et améliorées produites en côte d'Ivoire et au Nigéria (Enyisi et al., 2014 ; Deffan et al., 2015). Les glucides totaux sont des hydrates de carbone emmagasinés sous forme d'amidon dans les céréales. Selon Deffan et al. (2015), bien qu'ils constituent la principale source énergétique utilisable par l'organisme, ils interviennent également comme des laxatifs naturels et facilitent la digestion.

Les teneurs en protéines $(12,4$ et $12,6 \%$ MS) sont identiques à celles de certaines variétés de maïs (10,5-12,1\% MS) déterminées par Deffan et al. (2015). Ces teneurs en protéines sont supérieures à celles obtenues (4,5-9,9\% MS) par Enyisi et al. (2014). Selon Nuss et Tanumihardjo (2010), la teneur en protéines des céréales est très variable selon les types de variétés. Le rôle primordial d'une protéine alimentaire est de combler les besoins de l'organisme en azote et en acides aminés essentiels dont elle est composée.

Les teneurs de cendre obtenues $(1,6$ à $1,7 \%$ MS) sont comparables à ceux de Enyisi et al. (2014) qui ont trouvé respectivement des valeurs comprises entre 1,1 à $2,9 \%$ MS dans le maïs. La différence enregistrée au niveau des variétés s'expliquerait par le fait que les minéraux de maïs proviendraient de l'utilisation de la matière inorganique du sol, des applications des engrais et/ou au type de variété (Deffan et al., 2015).

Les teneurs en matière grasse obtenues dans les deux variétés de maïs frais bouilli sont très importants et contribueraient donc à la qualité nutritionnelle du produit. Ostlund et al. (2002) ont mentionné que les matière grasse de maïs sont très riche en acides gras polyinsaturés dont les phytostérols ont été les principaux composants responsables d'une diminution de l'absorption du cholestérol par l'organisme au cours d'une étude clinique chez l'humain.
Les composés phénoliques sont particulièrement présents chez les végétaux et ont la qualité d'antioxydants. Willcox et al. (2004) ont expliqué que les antioxydants sont des composés qui protègent les cellules du corps contre les radicaux libres. Ces derniers sont des molécules très réactives qui seraient responsables du développement des maladies cardiovasculaires, de certains cancers et d'autres maux relatifs au vieillissement. Donc ces teneurs en composés phénoliques contenus dans les grains de maïs frais bouilli seraient très bénéfiques pour la santé des consommateurs. Les composés phénoliques sont formés de trois groupes tels que les acides phénoliques, les flavonoïdes et les tanins (Dicko et al., 2006). La teneur en composés phénoliques de la variété jaune est significativement plus élevée au seuil de 5\% que celle de la variété blanche. LopezMartinez et al. (2009) et Chiremba et al. (2012) ont mentionné que la faible teneur en composés phénoliques confère aux variétés de maïs une bonne digestibilité des protéines, ce qui constitue donc un aspect positif pour la qualité nutritionnelle de cette céréale. Ainsi, le maïs de la variété blanche serait plus digeste que celui issu de la variété jaune.

Une bonne compréhension des relations existant entre les caractéristiques nutritionnelles, les propriétés technologiques et organoleptiques des variétés de maïs devrait permettre aux sélectionneurs de mieux orienter leurs programmes d'amélioration variétale d'une part, et aux utilisateurs de choisir les variétés en fonction de leur qualité technologique et nutritionnelle d'autre part.

\section{Conclusion}

Cette étude montre que la production des épis de maïs frais bouilli est une activité essentiellement féminine. Deux techniques de production sont en usage au Bénin, la cuisson du maïs frais avec les spathes et la cuisson sans les spathes. La qualité du maïs frais bouilli dépend de la variété et de la durée post-récolte. La variété jaune est apparue plus sucrée et plus appréciée que la variété blanche. Plus la durée post-récolte augmente, plus la qualité organoleptique du maïs frais bouilli diminue. Les caractéristiques nutritionnelles du maïs frais bouilli révèlent 
une richesse en glucides totaux, en protéines, en minéraux et composés phénoliques du produit, ce qui est bénéfique pour la santé.

\section{CONFLITS D'INTERETS}

Les auteurs déclarent qu'il n'y a aucun conflit d'intérêts.

\section{CONTRIBUTIONS DES AUTEURS}

Ce travail est une initiative de tous les auteurs. FH et JMK ont conçu le questionnaire d'enquête, réalisé les manipulations au laboratoire, analysé les données obtenues et ont écrit le manuscrit. JDH et MCN ont validé la fiche d'enquête, contribué à l'amélioration du manuscrit, validé les protocoles de laboratoire et supervisé tous les travaux.

\section{REFERENCES}

AOAC. 1995. Official Methods of Analysis of the Association of Official Analytical Chemists. Washington: AOAC.

Abadassi J. 2014. Characterization of traditional maize populations cultivated in Benin. International Journal of Biological and Chemical Sciences, 8(2): 434-442.

DOI: http://dx.doi.org/10.4314/ijbcs.v8i2.3

Adégbola PY, Aloukoutou A, Diallo B. 2011. Analyse de la compétitivité du maïs local au Bénin. PRESAO (Programme de Renforcement et Recherche sur la Sécurité Alimentaire en Afrique l'Ouest) Composante SRAI, Résumé N¹-201112-Maïs Http://Www.Aec.Msu.Edu/ Fs2/Presao.Htm (site consulté 10/12/2015)

Ba R, Monteiro NMF, Hounguè U, Donou Hounsodé MT, Gbaguidi F, BabaMoussa L. 2016. Perception des producteurs et impact des facteurs socioéconomiques sur la connaissance des mycotoxines du maïs en stockage au Bénin. International Journal of Biological and Chemical Sciences, 10(1): $\quad 155-166 . \quad$ DOI: http://dx.doi.org/10.4314/ijbcs.v10i1.11

Baco MN, Abdoulaye T, Sanogo D, Langyintuo A. 2010. Caractérisation des ménages producteurs de maïs en zone de savane sèche au Bénin, Rapport pays -
Enquête ménage - Benin. IITA Ibadan, Nigeria, $38 \mathrm{p}$.

Chiremba C, Taylor JRN, Rooney LW, Beta T. 2012. Phenolic acid content of sorghum and maize cultivars varying in hardness. Food Chemistry, 134: 81-88. DOI:10.1016/j.foodchem.2012.02.067

Deffan KP, Akanvou L, Akanvou R, Nemlin GJ, Kouamè PL. 2015. Évaluation morphologique et nutritionnelle de variétés locales et améliorées de maïs (zea mays 1.) produites en Côte d'Ivoire. Afrique Science, 11(3):181-196. http://www.afriquescience.info/documen t.php?id=4761.

Dicko MH, Gruppen H, Voragen AGJ, Van Berkel WJH. 2006. Biochemical characterization of major sorghum grain peroxidase. FEBS Journal, 273: 22932307. DOI: $10.1111 / \mathrm{j} .1742-$ 4658.2006.05243.x

Enyisi IS, Umoh VJ, Whong CMZ, Abdullahi IO, Alabi O. 2014. Chemical and nutritional value of maize and maize products obtained from selected markets in Kaduna State, Nigeria. African Journal of Food Science and Technology, 5(4): 100-104. DOI: http:/dx.doi.org/10.14303/ajfst.2014.029

FAO. 2013. FAO Statistical Yearbook 2013. FAO: Rome, Italy.

Hongbété F, Kindossi JM. 2017. Diversity and Traditional Consumption of Edible Insects in North Benin. Journal of Scientific Research and Reports, 14(1):111. DOI: $10.9734 / J S R R / 2017 / 32542$

Hongbété F, Tidjani A-K, Kindossi JM. 2017. Traditional Production Technology, Consumption And Quality Attributes Of Toubani: A Ready-To-Eat Legume Food From West Africa. African Journal of Biotechnology, 16(19): 1123-1130. DOI: 10.5897/AJB2017.15913

Houngbo NE. 2015. Diversité et critères d'adoption des cultivars de maïs (Zea mays L.) dans le village Zounnou, Centre Bénin. Journal of Applied Biosciences, 96: 9094-9101. DOI: http://dx.doi.org/10.4314/jab.v96i1.6

Kayodé APP, Hounhouigan DJ, Nout MJR, Niehof A. 2006. Household production of sorghum beer in Benin: Technological 
and socio-economical aspects. International Journal of Consumer Studies, 31, 258-264. DOI: 10.1111/j.1470-6431.2006.00546.x

Kindossi JM, Anihouvi VB, Vieira-Dalodé G, Akissoé NH, Jacobs A, Dlamini N, Pallet D, Hounhouigan DJ. 2012. Production, consumption, and quality attributes of Lanhouin, a fish-based condiment from West Africa. Food Chain, 2(1): 117-130. DOI: 10.3362/2046-1887.2012.009

Lopez-Martinez LX, Oliart-Ros RM, ValerioAlfaro G, Lee C-H, Parkin KL, Garcia HS. 2009. Antioxidant activity, phenolic compounds and anthocyanins content of eighteen strains of Mexican maize. LWT - Food Science and Technology, 42: 1187-92. DOI:10.1016/j.lwt.2008.10.010

Mestres C, Nago CM, Akissoe N, Matencio, F. 1997. End use quality of some african corn Kernels. 2. Cooking behavior of whole dry-milled maize flour; incidence of storage. Journal of Agricultural and Food Chemistry, 45: 565 -571.

Nago CM. 1997. La transformation alimentaire traditionnelle du maïs au Bénin: détermination des caractéristiques physico-chimiques des variétés en usage; relations avec l'obtention et la qualité des principaux produits dérivés. Thèse de doctorat d'Etat ES-Sciences, Université Paris7-Denis Diderot-UFR de Biochimie, $199 \mathrm{p}$.

Nuss ET, Tanumihardjo SA. 2010. Maize: A Paramount Staple Crop in the Context of Global Nutrition. Comprehensive Reviews in Food Science and Food Safety, 9:417-436. DOI: 10.1111/j.15414337.2010.00117.x

Ostlund RE, Jr, Racette SB, Okeke A, Stenson WF. 2002. Phytosterols that are naturally present in commercial corn oil significantly reduce cholesterol absorption in humans. American Journal of Clinical Nutrition, 75(6): 1000-1004.

PSRSA. 2010. Plan Stratégique de Relance du Secteur Agricole au Bénin : Orientations stratégiques et Plan d'action. Version finale, République du Bénin, Cotonou, 1110.

Sacca C, Adinsi L, Anihouvi V, Akissoé N, Dalodé G, Mestres C, Jacobs A, Dlamini N, Pallet D, Hounhouigan DJ. 2012. Production, consumption, and quality attributes of Akpan - a yoghurt-like cereal product from West Africa. Food Chain., 2(2): 207-220. DOI: 10.3362/2046-1887.2012.018

Sarr B, Kafando L, Atta S. 2011. Identification des risques climatiques de la culture du maïs au Burkina Faso. International Journal of Biological and Chemical Sciences, 5(4): 1659-1675. DOI: http://dx.doi.org/10.4314/ijbcs. v5i4.28

Semassa AJ, Padonou SW, Anihouvi VB, Akissoé NH, Adjanohoun A, BabaMoussa L. 2016. Diversité variétale, qualité et utilisation du maïs (Zea Mays) en Afrique de l'Ouest: Revue critique. European Scientific Journal, 12(18): 197-217. DOI: 10.19044/esj.2016. v12n18p197

Singleton, V. L. Orthofer R. and LamuelaRaventos R.M. 1999. Analysis of total phenols and other oxidant substrates and antioxidants by means of Folin-Ciocalteu reagent. Methods Enzymol., 299: 152178. DOI: https://doi.org/10.1016/ S0076-6879(99)99017-1

Sodjinou E, Adegbola PY, Zinsou J, Oloukoi L. 2008. Stratification des systèmes de production des filières riz et maïs au Bénin. PAPA/INRAB-ADRAO, 46p.

Tshite FN, Mulamba VT, Ndianabo MJT. 2015. Mise au point d'une farine précuite à base de maïs (Zea mays) et de soja (Glycine max) par la méthode traditionnelle. International Journal of Biological and Chemical Sciences, 9(6): 2608-26022. DOI: http://dx.doi.org/ 10.4314/ijbcs.v9i6.8

Willcox JK, Ash SL, Catignani GL. 2004. Antioxidants and prevention of chronic disease. Critical Reviews in Food Science and Nutrition, 44(4): 275-295. DOI: $10.1080 / 10408690490468489$. 